\title{
AN ASYMPTOTIC EXPANSION FOR THE GENERALISED QUADRATIC GAUSS SUM REVISITED
}

\author{
R. B. PARIS
}

Abstract. An asymptotic expansion for the generalised quadratic Gauss sum

$$
S_{N}(x, \theta)=\sum_{j=1}^{N} \exp \left(\pi i x j^{2}+2 \pi i j \theta\right),
$$

where $x, \theta$ are real and $N$ is a positive integer, is obtained as $x \rightarrow 0$ and $N \rightarrow \infty$ such that $N x$ is finite. The form of this expansion holds for all values of $N x+\theta$ and, in particular, in the neighbourhood of integer values of $N x+\theta$. A simple bound for the remainder in the expansion is derived. Numerical results are presented to demonstrate the accuracy of the expansion and the sharpness of the bound.

Mathematics subject classification (2010): 11L05, 41A30, 41A60, 41A80. Keywords and phrases: Quadratic Gauss sum, asymptotics, curlicues.

\section{REFERENCES}

[1] T. M. Apostol, Introduction to Analytic Number Theory, Springer, New York, 1984.

[2] E. A. Coutsias AND N. D. KaZARINoff, Disorder, renormalizability, theta functions and Cornu spirals, Physica 26D (1987), 295-310.

[3] E. A. COUTSIAS AND N. D. KAZARINOFF, The approximate functional formula for the theta function and Diophantine Gauss sums, Trans. Amer. Math. Soc. 350 (1988), 615-641.

[4] F. M. DEKKING AND M. MENDÈS-FRANCE, Uniform distribution modulo one: a geometrical viewpoint, J. Reine Angew. Math. 329 (1981), 143-153.

[5] R. Evans, M. Minei And B. YeE, Incomplete higher-order Gauss sums, J. Math. Anal. Appl. 281 (2003), 454-476.

[6] H. Fiedler, W. JURKAT ANd O. KÖRner, Asymptotic expansions of finite theta series, Acta Arithmetica 32 (1977), 129-146.

[7] G. H. Hardy and J. E. Littlewood, Some problems of Diophantine approximation. II: The trigonometrical series associated with the elliptic $\vartheta$-functions, Acta Math. 37 (1914), 183-236. Reprinted in Collected Papers of G.H. Hardy, Vol. 1, 67-112, Oxford University Press, Oxford.

[8] E. KRÄTZEL, Analytische Funktionen in der Zahlentheorie, Teubner, Stuttgart-Leipzig, 2000.

[9] D. H. LEHMER, Incomplete Gauss sums, Mathematika 23 (1976), 125-135.

[10] F. W. J. Olver, Asymptotics and Special Functions, Academic Press, New York, 1974. Reprinted A.K. Peters, Massachussets, 1997.

[11] F. W. J. Olver, D. W. Lozier, R. F. Boisvert and C. W. Clark (eds.), NiST Handbook of Mathematical Functions, Cambridge University Press, Cambridge, 2010.

[12] R. B. PARIS, An asymptotic expansion for the generalised quadratic Gauss sum, Appl. Math. Sci. 2 (2008), 577-592.

[13] F. Sullivan and U. Zannier, A note on incomplete quadratic Gauss sums, Boll. Un. Mat. Ital. A6 (1992), 49-57. 